\title{
Estudio del comportamiento de fundición CADI sometida a FCR
}

\section{Study of the Carbidic ADI performance under RCF condition}

\author{
Diego Iván Pedro ${ }^{1}$, Sebastián Laino ${ }^{1}$, Ricardo Cesar Dommarco ${ }^{1}$
}

\author{
${ }^{1}$ Grupo Tribología - Facultad de Ingeniería - Universidad Nacional de Mar del Plata - Div. Metalurgia - INTEMA - \\ CONICET CP: 7600, Mar del Plata, Buenos Aires, Argentina. \\ e-mail: dpedro@fi.mdp.edu.ar; slaino@fi.mdp.edu.ar; dommarco@fi.mdp.edu.ar
}

\section{RESUMEN}

La Fundición Nodular (DI) es un desarrollo en las fundiciones de hierro introducido en la decada del 50, con grafito libre de morfología nodular. La Fundición Nodular Austemperada (ADI) es un desarrollo posterior de DI, consistente en un tratamiento térmico posterior de Austemperado para obtener un material de alta resistencia y tenacidad. La Fundición Nodular Austemperada con Carburos (CADI) es un desarrollo más reciente de DI, modificada con la presencia de carburos estabilizados (partículas duras) con el objetivo de mejorar la resistencia al desgaste abrasivo aunque perdiendo tenacidad al impacto frente al ADI.

Hasta la actualidad, la investigación del CADI se ha basado principalmente en sus aplicaciones como material resistente a la abrasión. Su desempeño frente a otros mecanismos de desgaste no ha sido totalmente evaluado. Por lo tanto, por primera vez, en el presente trabajo se evalúa la resistencia a la fatiga de contacto por rodadura (FCR) del CADI relacionando su microestructura con la respuesta frente a las cargas cíclicas en los contactos puntuales o lineales.

Se fabricaron muestras en forma de disco mecanizando placas coladas y luego fueron tratadas térmicamente por austemperizado. Los ensayos de desgaste fueron realizados en una máquina de ensayos de FCR del tipo Arandela plana.

Los resultados muestran similar nodularidad y tamaño de nódulo para ADI y CADI pero el conteo nodular fue menor para el CADI.

La resistencia a FCR de CADI resultó superior a la de ADI teniendo ambos materiales durezas similares.

Palabras clave: CADI, RODADURA, CONTACTO, FATIGA

\section{ABSTRACT}

Ductile Iron (DI) is a development of the iron alloys introduced in the $50 \mathrm{~s}$, with free graphite in the form of nodules. Austempered Ductile Iron (ADI) is a development of DI later introduced, consisting on austempering heat treatment in order to obtain a high strength and high toughness material. Carbidic Austempered Ductile Iron (CADI) is a more recent development of DI, modified by the presence of stabilized carbides (hard particles) in order to improve its abrasion resistance but with lower impact toughness than ADI.

Until now, the research on CADI was mainly related with its application as an abrasion resistant material. Its performance facing other wear mechanisms has not been fully evaluated. Therefore, for the first time, in this paper CADI Rolling contact fatigue (RCF) resistance is evaluated in order to relate the microstructure to the response under cyclic loading in point or line contacts.

Samples were manufactured to a disk shape by machining cast plates and then were heat treated by austempering. The wear tests were performed in a flat washer type RCF tester.

The results show that both, ADI and CADI, have similar nodularity and nodule size but when the nodule count was lower for CADI.

The RCF resistance of CADI was higher than that of ADI even though both material variants had very simi- 
lar hardness.

Keywords: CADI, ROLLING, CONTACT, FATIGUE

\section{INTRODUCCIÓN}

La Fundición Nodular Austemperada (ADI) es una aleación de hierro con carbono que se procesa por colada, y que luego recibe un tratamiento térmico que le confiere una alta resistencia a la rotura y buena tenacidad al impacto (por encima de $1600 \mathrm{MPa}$ para el grado 5 y $100 \mathrm{~J}$ para correspondiente al grado 1, según ASTM A 834-95). La combinación de facilidad y economía de procesamiento, combinado con estas excelentes propiedades mecánicas, le permiten reemplazar a los aceros forjados en ciertas aplicaciones. El ADI también posee excelente resistencia al desgaste bajo diferentes mecanismos, como fatiga de contacto por rodadura (FCR), adhesión y abrasión [1,2]. Las aplicaciones típicas de este material son aquellas que requieran una combinación de buena resistencia a la rotura y tenacidad, a menudo combinado con una muy buena resistencia al desgaste, en piezas tales como, engranajes, levas, cigüeñales, piezas de suspensión, y piezas varias para sectores de la industria del transporte, agrícola, minera, metalmecánica, construcción, etc.

La evolución tecnológica relativa al desarrollo de la fundición nodular, desde su descubrimiento en la década de los '50s y hasta nuestros días, muestra en la última década un interés creciente en el estudio de una nueva variante de este material que incorpora carburos en su microestructura. La Fundición Nodular Austemperada con Carburos (CADI), es una variante del ADI de desarrollo reciente, que busca mejorar la resistencia al desgaste a través del refuerzo con carburos (partículas duras), resultando en una mejora en la resistencia a la abrasión aunque, por otro lado, disminuye la tenacidad al impacto [3]. No obstante, el CADI posee una tenacidad al impacto similar o mayor que otros materiales con resistencia al desgaste similar [4], pero un menor costo de producción. Además, permite la comercialización de un material con mayor valor agregado, con el "know how" que involucra el control de la microestructura determinando la composición química de colada y la selección de los parámetros de tratamiento térmico, sin involucrar una inversión de capital para incorporarlo a la oferta de las plantas de fundición actuales.

Hasta el presente, casi la totalidad de los trabajos relativos al CADI están relacionados con su aplicación como material resistente a la abrasión, siendo escasos los trabajos que evalúan otros mecanismos de desgaste y nulos en lo relativo a FCR. Esto resulta llamativo siendo que son numerosas las aplicaciones de las fundiciones de hierro atruchadas (que combinan en su microestructura la presencia de grafito libre, ya sea laminar o nodular, con carburos), que son sometidas a condiciones de desgaste por fatiga de contacto y/o deslizamiento, como es el caso de levas y seguidores de leva de la industria (automotriz, metalmecánica, agrícola), y rodillos de laminación de la industria de los polímeros.

Por lo tanto, siendo que el CADI es una variante del ADI que se encuentra en desarrollo y cuyo abanico de aplicaciones sigue en exploración, en el presente trabajo se estudia su comportamiento frente al desgaste por FCR, evaluando las variables de procesamiento que afectan su microestructura por lo tanto su resistencia al desgaste. La resistencia a FCR en principio está relacionada con la dureza del material, la que puede modificarse con la cantidad de carburo, y las características de los nódulos de grafito y de la matríz ausferrítica (cantidad de austenita y su contenido de carbono). Esto se puede controlar a través de la composición química, el diseño del molde, el tratamiento térmico, etc.

La adición de Cromo a la Fundición Nodular (FN) produce un estrechamiento de la ventana de temperatura existente en la transformación eutéctica [5], entre los diagramas Fe-C estable y meta-estable, Fig. 1-a. Este efecto, combinado con la micro-segregación del Cr, resulta en la precipitación de carburos ledeburíticos aleados con cromo, debido a la participación de la transformación metaestable durante la solidificación, Fig. 1-b. Bajo estas condiciones se obtiene fundición nodular con carburos directos de la solidificación, resultando en una microestructura con grafito libre y carburos, denominada Fundición Nodular Atruchada (FNA), luego de la transformación en estado sólido correspondiente al tratamiento térmico de austemperado, este material es transformado en CADI.

Por lo tanto, en el presente trabajo se colaron muestras de FNA aleada con Cr y posteriormente fueron tratadas térmicamente por austemperado para obtener CADI. Mediante el mecanizado posterior se obtuvieron probetas para caracterizar microestructura, dureza y resistencia a FCR. 


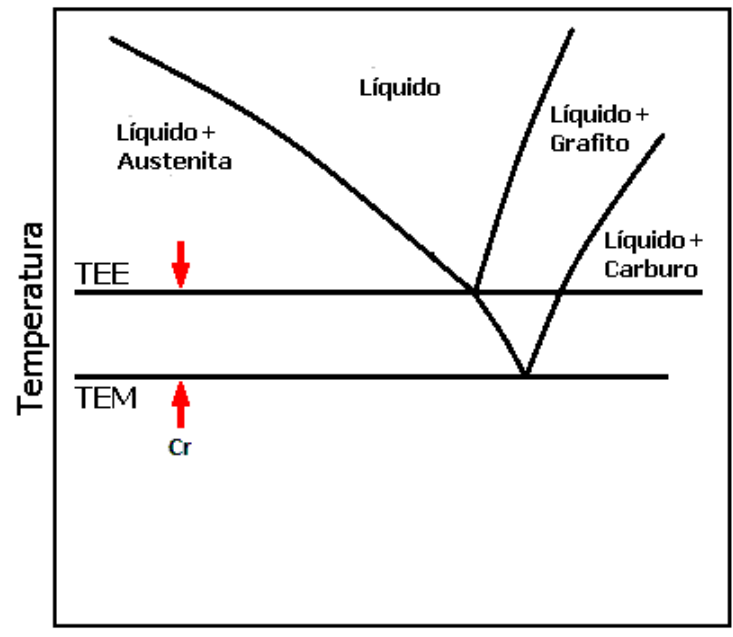

Carbono Equivalente

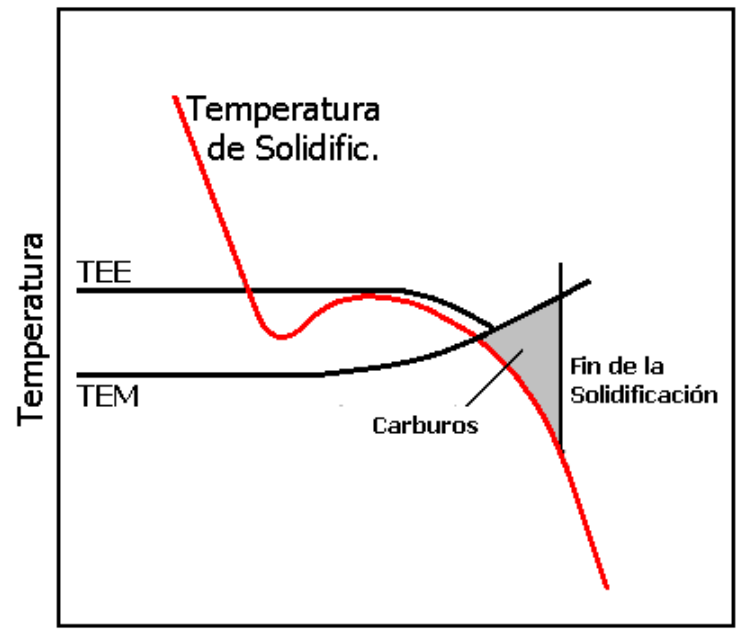

Tiempo

Figura 1: a) Influencia del Cromo sobre la temperatura de la transformación eutéctica, tanto para el diagrama Fe-C estable (TEE) como metaestable (TEM), b) Efecto combinado de la microsegregación sobre las temperaturas TEE y TEM superpuesto con la temperatura durante la solidificación, causando la precipitación de carburos ledeburíticos.

\section{MATERIALES Y MÉTODOS}

\subsection{Material y preparación de Muestras}

La Fundición Nodular (FN) y Nodular Atruchada (FNA) utilizadas en este trabajo fueron fabricadas en un horno de inducción que funciona con frecuencia de red y posee una capacidad de $1500 \mathrm{~kg}$. La inoculación y nodulización se realizó con FeSi y FeSiMg, respectivamente, aplicando técnicas convencionales, en una cuchara de $500 \mathrm{~kg}$ con bolsillo de nodulización. El material se obtuvo en forma de placas prismáticas de (250x250x13) $\mathrm{mm}$.

Las probetas para ensayos de fatiga de contacto, fueron obtenidas a partir de las placas de FN y FNA, por mecanizado en forma de discos de $\sim 60 \mathrm{~mm}$ de diámetro, $\sim 10 \mathrm{~mm}$ de espesor y un agujero central, y luego se realizó el tratamiento térmico a través de diferentes austemperizados resultando en diferentes grupos de muestras con microestructuras disímiles. La FNA fue austenizada a $T_{\gamma}=910{ }^{\circ} \mathrm{C}$ por $t_{\gamma}=90$ min y luego austemperada en baño de sales a $T_{a}=280{ }^{\circ} \mathrm{C}$ y $360^{\circ} \mathrm{C}$ durante $t_{a}=90 \mathrm{~min}$. La FN fue austenizada a $T_{\gamma}=910^{\circ} \mathrm{C}$ por $t_{\gamma}=90 \mathrm{~min}$ y luego austemperada en baño de sales a $T_{a}=280{ }^{\circ} \mathrm{C}$ por $t_{a}=90 \mathrm{~min}$, fue utilizada como testigo para comparar el comportamiento a FCR. Las muestras fueron desbastadas utilizando una rectificadora de bandera para remover cualquier influencia superficial por el tratamiento térmico y luego pulido manualmente con papel abrasivo al agua grano 100.

Tabla 1: Identificación, material y parámetros de tratamiento térmico.

\begin{tabular}{c|c|c|c|c|c}
\hline IDENT. & MATERIAL & $\begin{array}{c}\text { TEMP. AUSTENIZ, } \\
\mathbf{T}_{\boldsymbol{\gamma}}-\mathbf{o} \mathbf{C}\end{array}$ & $\begin{array}{c}\text { TIEMPO AUSTENIZ, } \\
\mathbf{t} \boldsymbol{\gamma}-\mathbf{m i n}\end{array}$ & $\begin{array}{c}\text { TEMP AUSTEMP, } \\
\text { Ta - } \mathbf{~} \mathbf{C}\end{array}$ & $\begin{array}{c}\text { TIEMPO AUS- } \\
\text { TEMP, } \mathbf{t a}-\mathbf{m i n}\end{array}$ \\
\hline ADI280 & FN & 910 & 90 & 280 & 90 \\
\hline CADI280 & FNA & 910 & 90 & 280 & 90 \\
\hline CADI360 & FNA & 910 & 90 & 360 & 90 \\
\hline
\end{tabular}

La composición química del material (\% en peso) fue analizada mediante espectrometría de emisión óptica con excitación por chispa, con un espectrómetro marca Baird, con una fuente de excitación DV6. Los valores reportados surgieron del valor promedio de al menos 4 determinaciones.

Las muestras fueron preparadas para metalografía utilizando técnicas convencionales para el corte, desbaste y pulido manual. El ataque se realizó con el reactivo Nital $2 \%$. Para evaluar la nodularidad (caracterización de la morfología del grafito y el conteo nodular se utilizó la norma ASTM A247, mientras que para cuantificar los porcentajes en volumen, tanto de grafito como de carburo, se utilizó un analizador de imágenes (Image-Pro Plus). La caracterización fue realizada sobre la superficie de las probetas, pulidas y fotogra- 
fiadas. Los carburos fueron revelados mediante el ataque químico con persulfato de amonio en solución acuosa (10\%) durante 2 min, luego fotografiado en microscopio para el análisis de imagen.

La dureza se midió mediante el método Rockwell, utilizando la escala C (HRC) con un durómetro de banco. También se midió dureza Vickers a escala micro de los diferentes microconstituyentes, utilizando cargas de 200 g y 500 g en un microdurómetro de banco. La dureza se reporta en promedio y desviación estándar de al menos 5 determinaciones.

\subsection{Ensayos de Fatiga de Contacto por Rodadura}

Las probetas fueron ensayadas en una máquina tipo arandela plana (1), de acuerdo al esquema de la Fig. 2. Se utiliza como contra-muestra un rodamiento de empuje axial tipo 51107 (2) montado sobre una rótula (3) con el objetivo de minimizar cualquier anomalía en la distribución de la carga proveniente de la desalineación del plano de la muestra con respecto al plano que contiene la trayectoria de las bolillas. Se utilizó una velocidad de rotación de la muestra de $1748 \mathrm{rpm}$ resultando en una frecuencia de carga de $1,1 \times 10^{6}$ ciclos por hora.

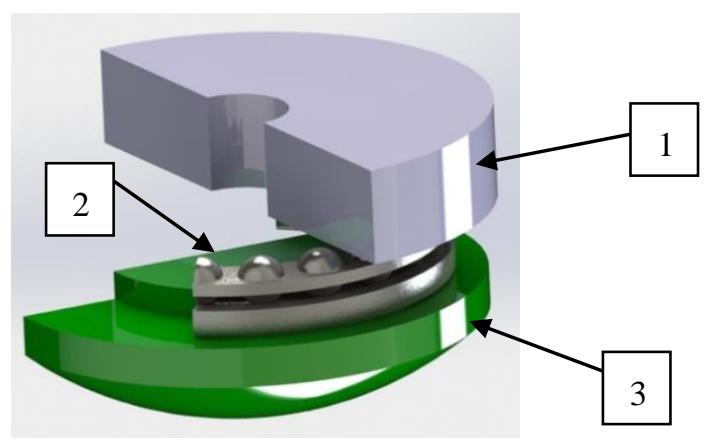

Figura 2: Disposición de los principales elementos en el ensayo de FCR, 1) probeta, 2) rodamiento de empuje axial tipo 51107 (contramuestra), 3) rótula para compensar posible desalineación.

La carga de trabajo se eligió para una relación $p_{0} / k_{k} \sim 4$, donde “ $k_{k}$ " es el límite elástico cinemático, resultando en presión de contacto $p_{0} \sim 1800 \mathrm{MPa}$, por bolilla [6,7].

La lubricación es por inmersión en una cuba con aceite ISO VG 100, Tellus de Shell, con filtración magnética.

Se utilizó un espesor de película lubricante específico $1<\lambda<3$, donde $\lambda$ es el cociente entre el mínimo espesor de película lubricante, $\mathrm{h}_{0}$ y la rugosidad superficial compuesta $\sqrt{\sigma_{A}^{2}+\sigma_{B}^{2}}$, donde $\sigma_{A, B}$ son las rugosidades superficiales de las bolillas y la muestra respectivamente. Se reporta la rugosidad superficial promedio de al menos 4 mediciones. Fue medida con un rugosímetro Taylor Hobson Surtronic 3+ con punta de diamante y utilizando un cut off de $0,8 \mathrm{~mm}$. La rugosidad superficial resultó en un $\mathrm{Ra}=0,33 \pm 0,05 \mu \mathrm{m}$ para las muestras de ADI 280, $\mathrm{Ra}=0,26 \pm 0,05 \mu \mathrm{m}$ para las de CADI360 y de $\mathrm{Ra}=0,36 \pm 0,05 \mu \mathrm{m}$ para las muestras de CADI280. Tanto $p_{0}$ como $\mathrm{h}_{0}$ fueron calculadas teóricamente con la teoría de contacto ideal elástico de Hertz y el cálculo de lubricación elastohidrodinámica de Hamrock y Dowson [8]. El espesor mínimo de película lubricante calculado fue de $h_{0}=0,6 \mu \mathrm{m}$ determinando el espesor de película específico en $\lambda=1,8$; 2,3; 1,7 respectivamente. La carga aplicada genera un diámetro de contacto ideal $2 \mathrm{a} \sim 170 \mu \mathrm{m}$.

La máquina cuenta con un sensor de vibraciones que detiene el ensayo cuando se alcanza la falla. El criterio de falla fue establecido en correspondencia con el nivel de vibración al momento en el cual se observa un cráter cuyo tamaño alcance el ancho del camino de rodadura. La vida hasta la falla se midió con un contador de horas, y esta cantidad luego fue convertida a ciclos de carga.

Se realizó un mínimo de 6 ensayos para cada variante de material, analizando los resultados estadísticamente y ajustándolos con una distribución de 2 parámetros de Weibull. Estos últimos son $\eta$ y $\beta$ y determinan la posición de la recta resultante del ajuste sobre el gráfico, representan la vida esperada para una probabilidad de falla de 63,3\% (posición) y la forma de la distribución (pendiente), respectivamente.

\section{RESULTADOS}

\subsection{Caracterización Metalográfica}

Los resultados de los análisis de composición química para la FN y la FNA se listan en la Tabla 2. En la FN, 
la composición resultó aproximadamente eutéctica, por el contrario, la FNA resultó hipereutéctica.

Tabla 2: Composición química de la FN y FNA (Luego del tratamiento térmico ADI y CADI respectivamente) (wt \%).

\begin{tabular}{l|l|l|l|l|l|l|l|l|l|l|l|l}
\hline & $\mathbf{F e}$ & $\mathbf{C}$ & $\mathbf{C r}$ & $\mathbf{S i}$ & $\mathbf{M n}$ & $\mathbf{S}$ & $\mathbf{P}$ & $\mathbf{M g}$ & $\mathbf{N i}$ & Mo & $\mathbf{C u}$ & $\mathbf{C E}$ \\
\hline FN/ADI & Bal. & 3,43 & - & 2,68 & 0,5 & $<0,04$ & $<0,04$ & 0,05 & 0,62 & 0,44 & 0,97 & 4,26 \\
\hline FNA/CADI & Bal. & 3,66 & 1,53 & 3,01 & 0,42 & $<0,02$ & $<0,04$ & $<0,02$ & 0,239 & 0,313 & 0,875 & 4,5 \\
\hline
\end{tabular}

La Fig. 3-a muestra la microestructura de la FN, para la cual la nodularidad resultante fue mayor al $80 \%$, el conteo nodular $(\mathrm{CN}) \sim 320 \mathrm{nod} / \mathrm{mm}^{2}$ y el diámetro promedio de los nódulos (DPN) de $\sim 18 \mu \mathrm{m}$ (corresponde a $\mathrm{N}^{\circ} 6$, ASTM A247). El contenido de grafito resultó de $\sim 8,6 \%$. La FNA, Fig. 3-b, tuvo una nodularidad mayor al $80 \%, \mathrm{CN}$ de $\sim 193 \mathrm{nod} / \mathrm{mm}^{2}$ y un DPN de $\sim 21 \mu \mathrm{m}$ (corresponde a No6, ASTM A247). El contenido en volumen de grafito resultó de $\sim 6,1 \%$ y el contenido en volumen de carburos de $6,4 \%$ (Fig. 4). En ambos casos la matriz resultó perlítica, no obstante, se observa una estructura más fina en la FN y, a su vez, algunas pequeñas regiones ferríticas. En ambos casos se describe la microestructura directa de colada, luego de tratarlos térmicamente nos referimos a la FN y a la FNA como ADI y CADI respectivamente según la Tabla 1.

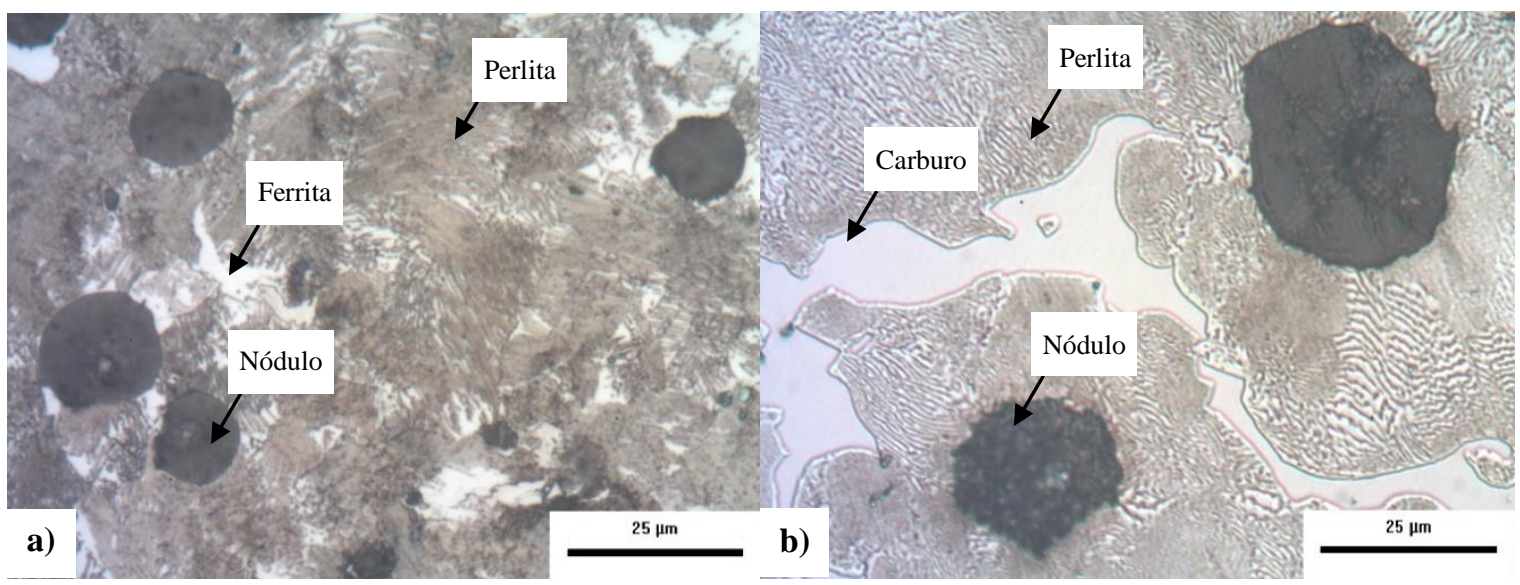

Figura 3: Microestructuras en bruto de colada, a) FN, nódulos de grafito inmersos en una matriz de perlita fina y algo de ferrita, b) FNA, nódulos de grafito inmersos en una matriz de perlita gruesa+carburos. Nital $2 \%$.

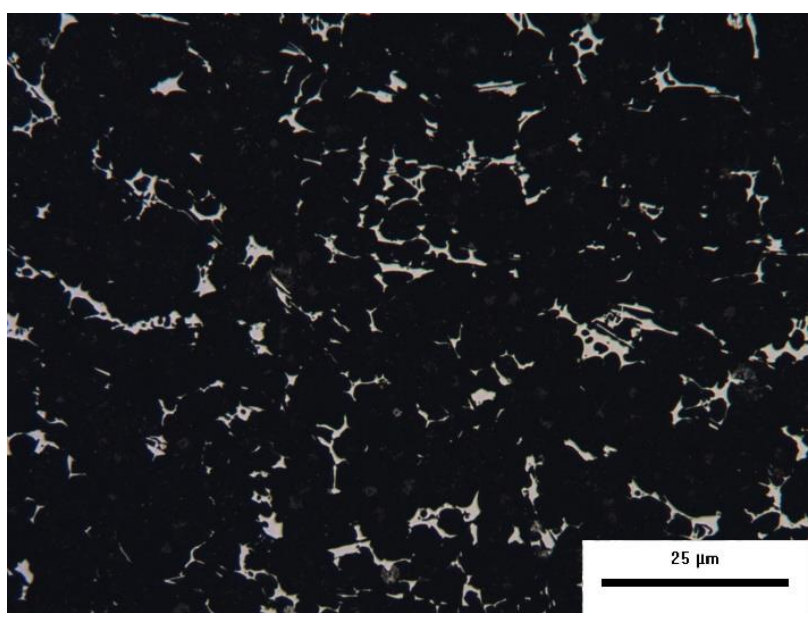

Figura 4: Captura de microscopio óptico utilizada para el conteo de Carburos. Ataque de CADI, 2 min en solución acuosa de persulfato de amonio $10 \%$.

Las microestructuras de las muestras CADI280 y CADI360 se presentan en la Fig. 5. Se observan nódulos de grafito y carburos no disueltos durante el tratamiento térmico debido a su estabilización con elemen- 
tos de aleación [3]. La variante CADI280, Fig. 5-a, posee una estructura compuesta por una mezcla cerrada de ferrita acicular y austenita retenida denominada ausferrita, correspondiente a una bainita inferior. En la variante de CADI360, Fig. 5-b, se observa una ausferrita más gruesa y abierta, de aspecto plumoso, se corresponde con una bainita superior.

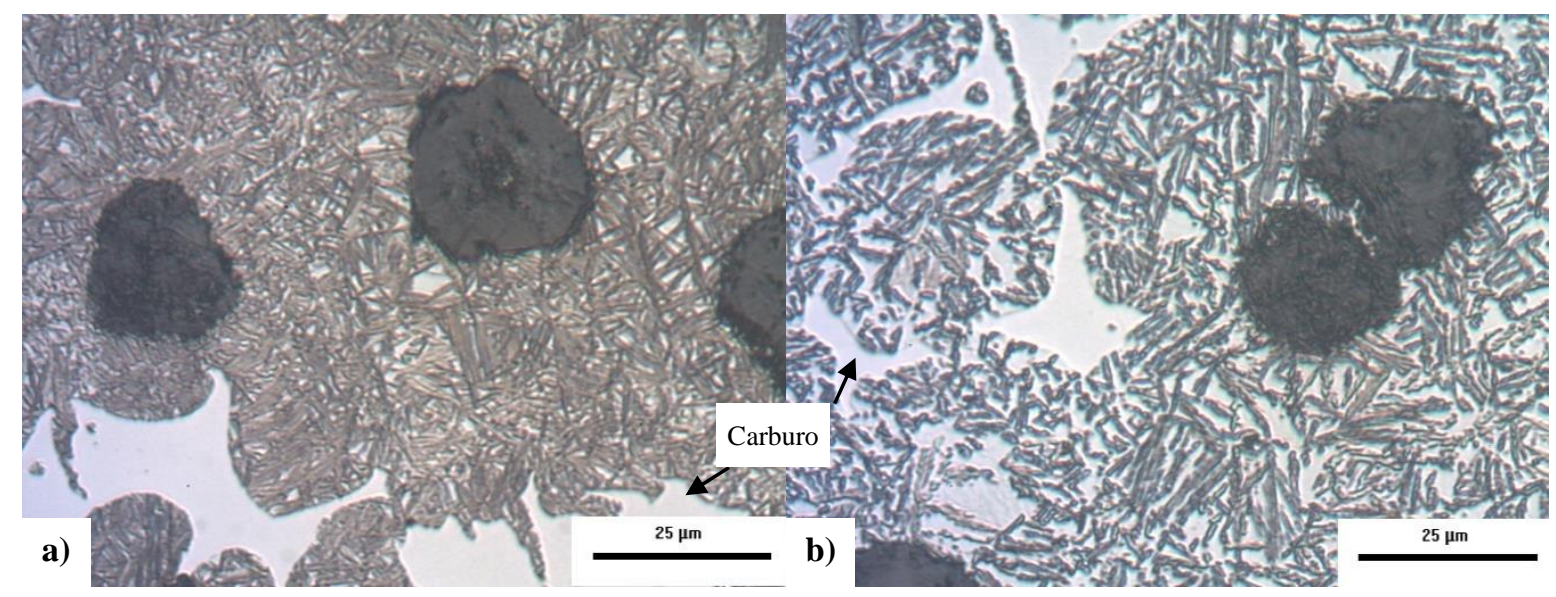

Figura 5: Microestructuras correspondientes de CADI, a) $\mathrm{Ta}=280$, b) $\mathrm{Ta}=360 . \mathrm{Nital} 2 \%$.

La Fig. 6 corresponde a la microestructura de la variante ADI280, donde se observan nódulos de grafito inmersos en una matriz de ausferrita inferior, predominantemente acicular.

La dureza promedio de las muestras de ADI280 resultó de 44,8 HRC, mientras que las de CADI360 y CADI280 resultaron de 44,8 y 50,2, respectivamente. A su vez, se resume el rango de dureza de las diferentes fases de cada variante en la tabla 3, observadas por micro-indentación. Se reportan rangos ya que dentro de la microestructura se observan diferentes regiones en relación con la presencia de micro-segregación.

Tabla 3: Dureza medida por micro-indentación sobre las diferentes fases y dureza global para cada material.

\begin{tabular}{l|l|l|l}
\hline MUESTRA & MATRIZ $(\mathbf{H V})$ & CARBUROS $(H V)$ & GLOBAL $(\mathbf{H R C})$ \\
\hline ADI280 & $499(\sigma=41)$ & - & $44,8(\sigma=0,4)$ \\
\hline CADI360 & $451(\sigma=48)$ & $989(\sigma=64)$ & $44,8(\sigma=1)$ \\
\hline CADI280 & $508(\sigma=52)$ & $978(\sigma=82)$ & $50,2(\sigma=1,3)$ \\
\hline
\end{tabular}

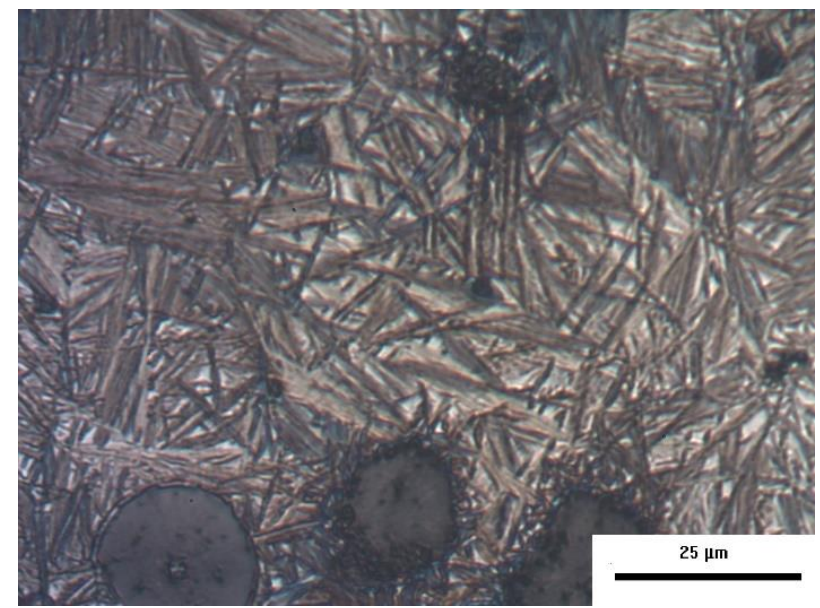

Figura 6: Microestructura correspondiente a ADI280

\subsection{Ensayos de Fatiga de Contacto}

La Fig. 7 muestra los resultados obtenidos, analizados mediante el cálculo estadístico de dos parámetros de Weibull, graficando la Probabilidad de Falla versus el Número de Ciclos de Carga. Los parámetros fueron 
calculados utilizando Rango de Mediana para la determinación de las probabilidades asociadas a los datos y luego aplicando mínimos cuadrados. Resulta apropiado para ajustar muestras de tamaño reducido (2-100 resultados). Se calculó el $r^{2}$ de las curvas ajustadas a los datos resultando en los tres casos en ajustes buenos $\left(r^{2}>0,925\right)$. En la tabla 4 se listan los valores característicos de las distribuciones de Weibull correspondientes a cada variante de material, junto con algunas características micro-estructurales y de lubricación.

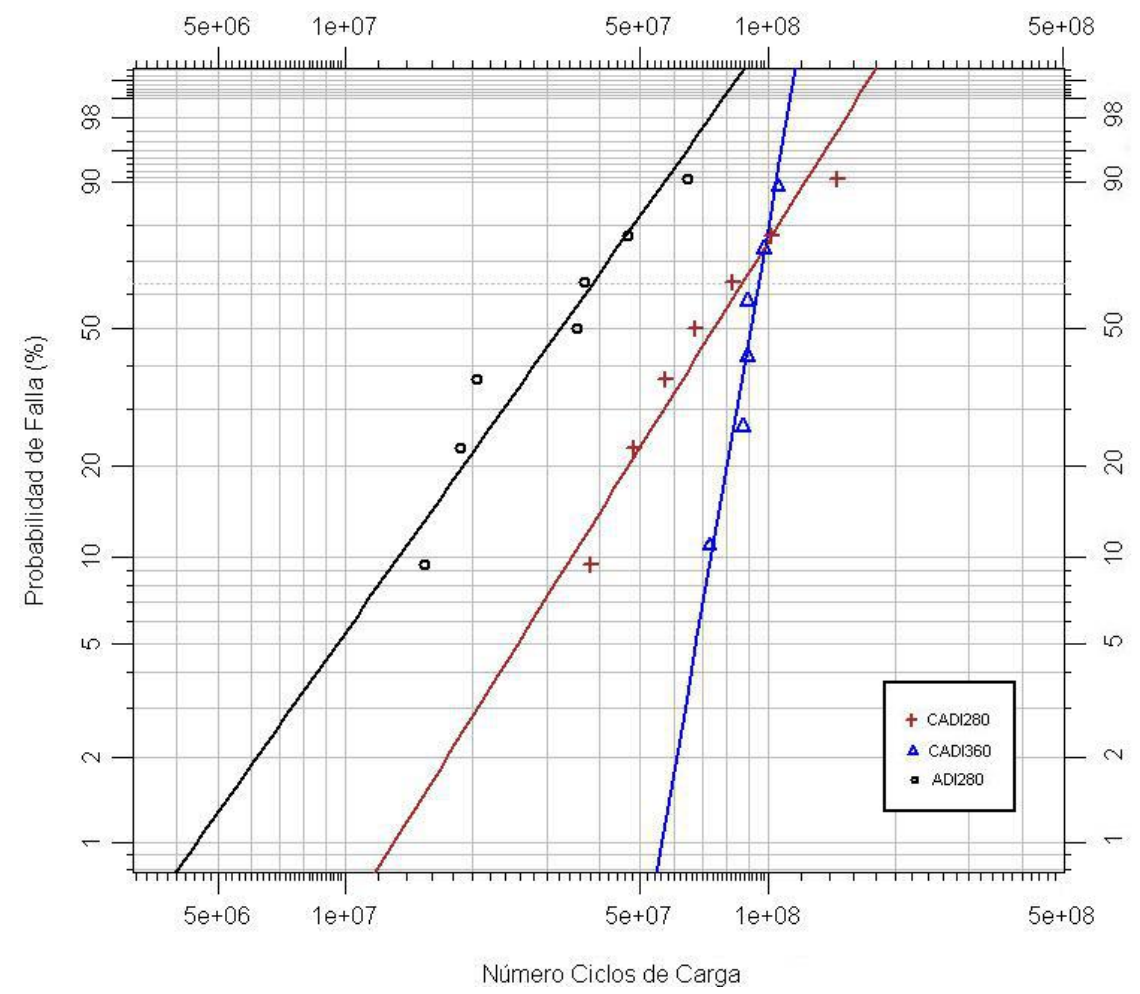

Figura 7: Diagrama de Weibull de 2 parámetros.

Tabla 4: Valores característicos de las curvas Weibull correspondientes a los ensayos FCR.

\begin{tabular}{l|l|l|l|l|l|l|l|l|l}
\hline MUESTRA & $\lambda$ & HRC & CN & DPN - $\boldsymbol{~}$ m & \%GRAF & \%CARB & L $_{50}$ & $\eta\left(\mathrm{L}_{63,3}\right)$ & $\boldsymbol{\beta}$ \\
\hline ADI280 & 1,8 & 44,8 & 320 & 18 & 8,6 & 0 & 32,38 & 38,45 & 2,134 \\
\hline CADI360 & 2,3 & 44,8 & 193 & 21 & 6,1 & 6,4 & 91,21 & 95,13 & 8,719 \\
\hline CADI280 & 1,7 & 50,2 & 193 & 21 & 6,1 & 6,4 & 74,76 & 86,96 & 2,425 \\
\hline
\end{tabular}

\section{DISCUSIÓN}

En los gráficos de Weibull de la Fig. 7, se aprecia que la variante CADI360 posee la mayor resistencia a la FCR, seguida por la CADI280, mientras que la variante de material de referencia, ADI280, resultó la de menor vida para las condiciones ensayadas.

Siendo los $\lambda \sim 2$ para todos los casos, se consideran los ensayos en igualdad de condiciones de lubricación. Dado que el proceso de falla por FCR está relacionado con la acumulación de micro-plasticidad, es de esperar que la dureza del material juegue un rol preponderante en la vida hasta la falla. Sin embargo, en el presente estudio, se observa que, si bien las variantes de material ADI280 y CADI360, poseen durezas similares, las segundas poseen una vida hasta la falla por FCR superior, por lo que es de esperar que otros elementos deban ser considerados para justificar este comportamiento, por supuesto, las características microestructurales.

Por ejemplo, se observa que el conteo nodular resultó mucho menor en las muestras CADI ya que parte del carbono pasa a formar los carburos, sin embargo manteniendo aproximadamente el tamaño de los nódulos, lo que se ve reflejado en el contenido de grafito. Con respecto a la iniciación de la falla, los nódulos que aparecen en la superficie de ensayo funcionan como defectos superficiales incrementando las tensiones de contacto [9]. La presencia de nódulos abiertos, o cercanos a la superficie, hace a estos materiales poco 
sensibles a los defectos extrínsecos como indentaciones, marcas de mecanizado, etc., resultando en una baja resistencia a la nucleación de la falla, como fue observado por Dommarco, et al. en ADI [7]. Siendo que el tamaño de los nódulos es similar en todos los casos, la probabilidad o velocidad de iniciación de la falla es mayor para las muestras de más alto $\mathrm{CN}$ (mayor cantidad de nódulos que actúan como defectos superficiales).

Por otro lado, existe evidencia experimental de la despreciable influencia del conteo nodular y el tamaño de nódulo sobre la velocidad de propagación de fisura por fatiga [10], por lo tanto, el efecto del conteo nodular sobre la propagación de la falla es despreciado.

Por otro lado, las probetas de CADI360 muestran una vida ligeramente superior a las de CADI280 en contraposición con sus durezas. Dado que el mecanismo de daño de FCR está íntimamente relacionado con la fatiga, podríamos atribuir dicho comportamiento en concordancia con lo observado por Tayanc, et al. [11]. Concluyeron que la ferrita de aspecto plumoso correspondiente a una bainita superior resulta en un mayor límite de fatiga que una ferrita acicular dentro de la microestructura ausferritica. Es decir, el CADI360 con una matriz de bainita superior resultó tener mejor desempeño al CADI 280 cuya matriz de bainita inferior contiene ferrita de morfología acicular.

\section{CONCLUSIONES}

La introducción de carburos a la microestructura resultó ser una forma eficaz de eliminar grafito libre reduciendo el conteo nodular. De esta forma, la cantidad de defectos superficiales, nódulos abiertos a la superficie, se reduce.

Los resultados obtenidos indican una mayor resistencia a FCR del CADI frente al ADI. Ello posibilita una nueva gama de aplicaciones para el material.

El presente trabajo inicia la investigación del material bajo este mecanismo de desgaste, analizando la vida a FCR y la relación de la microestructura con el mecanismo de desgaste, sin embargo la profundización de la cuestión requiere más investigación.

\section{AGRADECIMIENTOS}

Se reconoce al CONICET y a la Universidad Nacional de Mar del Plata por haber brindado todo su apoyo, equipos, instalaciones y financiamiento.

\section{BIBLIOGRAFÍA}

[1] MAGALHÃES, L., SEABRA, J. "Wear and scuffing of austempered ductile iron gears", Wear, v. 215, pp. 237-246, Marzo 1998.

[2] DOMMARCO,R., GALARRETA, I., ORTIZ, H., et al., "The use of ductile iron for wheel loader bucket tips", Wear, v. 249, pp. 101-108, Abril 2001.

[3] LAINO, S., SIKORA, J., DOMMARCO, R. "Development of wear resistant carbidic austempered ductile iron (CADI)", Wear, v. 265, pp. 1-7, Junio 2008.

[4] HAYRYNEN, K., BRANDENBERG, K. "Carbidic Austempered Ductile Iron (CADI) - The New Wear Material", Transactions of the American Foundry Society, v. 111, pp. 845-850, 2003.

[5] GUNDLACH, R.B., JANOWAK,J.F., BECHET, S. "On the problems with carbide formation in gray cast iron”, In: Materials Research Society Sumposium Proceedings, v. 34, pp. 251-261, 1985.

[6] DOMMARCO, R., SALVANDE, J. "Contact fatigue resistance of austempered and partially chilled ductile irons", Wear, v. 254, pp. 230-236, Febrero 2003.

[7] DOMMARCO, R., BASTIAS, P., DALL'O, H., et al., "Rolling Contact Fatigue (RCF) resistance of Austempered Ductile Iron (ADI)", Wear, v. 221, pp. 69-74, Octubre 1998.

[8] HAMROCK, B., DOWSON, D. "Isothermal elasto-hydrodynamic lubrication of point contacts. Part III. Fully flooded results", Journal of Tribology, Transactions of ASME F, v.99, n.2, pp. 264-276, Abril 1977.

[9] GUPTA,V., HAHN, G., BASTIAS, P., et al., "Influence of indent geometry on repeated twodimensional rolling contact", Journal of Tribology, v. 117, pp. 655-659, Octubre 1995.

[10] GRENO,G., OTEGUI,J., BOERI, R.“, Mechanisms of fatigue crack growth in Austempered Ductile Iron”, International Journal of Fatigue, v. 21, pp.35-43, Enero 1999.

[11] TAYANC, M., AZTEKIN,K., BAYRAM, A. "The effect of matrix structure on the fatigue behavior of austempered ductile iron”, Materials \& Design, v. 28, pp. 797-803, 2007. 